\title{
Overall Efficacy and Safety of Safinamide in Parkinson's Disease: A Systematic Review and a Meta-analysis
}

\author{
Riccardo Giossi ${ }^{1,2}$ (D) . Federica Carrara ${ }^{1} \cdot$ Martina Mazzari ${ }^{1} \cdot$ Francesco Lo Re $^{1,3} \cdot$ Michele Senatore $^{1,4}$. \\ Azzurra Schicchi ${ }^{5} \cdot$ Federica Corrù $^{1} \cdot$ Veronica Andrea Fittipaldo ${ }^{6} \cdot$ Arianna Pani $^{1} \cdot$ Irene Tramacere $^{7}$. \\ Antonio Emanuele Elia ${ }^{8} \cdot$ Francesco Scaglione $^{1,4}$
}

Accepted: 5 February 2021 / Published online: 5 March 2021

(c) The Author(s) 2021

\begin{abstract}
Background and Objective Safinamide is a novel anti-parkinsonian drug with possible anti-dyskinetic properties. Parkinson's disease (PD) is a complex disease. The objective of this systematic review and meta-analysis is to evaluate the efficacy and safety of safinamide administration compared to placebo in PD patients on multiple outcomes.

Methods PubMed, EMBASE, Cochrane CENTRAL, LILACS, and trial databases were searched up to 23 December 2020 for randomized controlled studies (RCTs) comparing safinamide to placebo, alone or as add-on therapy in PD. Data were extracted from literature and regulatory agencies. Primary outcomes were ON-time without troublesome dyskinesia, OFFtime, and Unified Parkinson's Disease Rating Scale (UPDRS) section III (UPDRS-III). Secondary outcomes included any dyskinesia rating scale (DRS), ON-time with troublesome dyskinesia, UPDRS-II, and Parkinson's Disease Questionnaire 39 (PDQ-39). In order to estimate mean difference (MD) and odds ratios with 95\% confidence intervals (CI), generic inverse variance and Mantel-Haenszel methods were used for continuous and dichotomous variables, respectively. Analyses were performed grouping by PD with (PDwMF) or without (PDwoMF) motor fluctuations, safinamide dose, and concomitant dopaminergic treatment. Summary of findings with GRADE were performed.

Results Six studies with a total of 2792 participants were identified. In PDwMF patients, safinamide $100 \mathrm{mg}$ as add-on to levodopa (L-dopa) significantly increased ON-time without troublesome dyskinesia (MD $=0.95 \mathrm{~h} ; 95 \% \mathrm{CI}$ from 0.41 to 1.49 ), reduced OFF-time ( $\mathrm{MD}=-1.06 \mathrm{~h}$; $95 \% \mathrm{CI}$ from -1.60 to -0.51 ), and improved UPDRS-III (MD $=-2.77 ; 95 \% \mathrm{CI}$ from -4.27 to -1.28 ) with moderate quality of evidence. Similar results were observed for the $50 \mathrm{mg}$ dose. However, the quality of evidence was moderate only for ON-time without troublesome dyskinesia, whereas for OFF-time and UPDRS-III was low. In PDwoMF patients taking a single dopamine agonist, safinamide $100 \mathrm{mg}$ resulted in little to no clinically significant improvement in UPDRS-III (MD $=-1.84 ; 95 \% \mathrm{CI}$ from -3.19 to -0.49 ), with moderate quality of evidence. Conversely, in PDwoMF patients, the $200 \mathrm{mg}$ and $50 \mathrm{mg}$ doses showed nonsignificant improvement in UPDRS-III, with very low and moderate quality of evidence, respectively. In PDwMF patients taking safinamide $100 \mathrm{mg}$ or $50 \mathrm{mg}$, nonsignificant differences were observed for ON-time with troublesome dyskinesia and DRS, with high and low quality of evidence, respectively. In the same patients, UPDRS-II was significantly improved at the $100 \mathrm{mg}$ and $50 \mathrm{mg}$ dose, with high and moderate quality of evidence. In PDwoMF, UPDRS-II showed a little yet significant difference only at $100 \mathrm{mg}$, with low quality of evidence. PDQ-39 resulted significantly improved only with the $100 \mathrm{mg}$ dose in PDwMF, with low quality of evidence.

Conclusion Overall, safinamide is effective in PDwMF patients taking L-dopa both at 100 and $50 \mathrm{mg}$ daily. Evidence for efficacy in early PD is limited. Further trials are needed to better evaluate the anti-dyskinetic properties of safinamide.
\end{abstract}

Riccardo Giossi

riccardo.giossi@unimi.it

Extended author information available on the last page of the article 


\section{Key Points}

Safinamide $100 \mathrm{mg}$ and $50 \mathrm{mg}$ daily is effective as add-on to L-dopa in improving ON-time without troublesome dyskinesia, OFF-time, UPDRS-III, UPDRS-II, and PDQ-39 in Parkinson's disease patients with motor fluctuations.

In patients with motor fluctuations, ON-time with troublesome dyskinesia and DRS were not significantly different between safinamide and placebo, with limited evidence. Dyskinesia should be better investigated as a primary outcome in future studies.

Safinamide showed little to no difference in improving UPDRS-III in non-fluctuating patients taking a dopamine agonist.

\section{Introduction}

Parkinson's disease (PD) is a neurodegenerative movement disorder that presents with rigidity, bradykinesia, and rest tremor. In 2015, PD prevalence has increased by $15.7 \%$ compared to 1990, and by 2040 the number of people affected by PD is projected to exceed 12 million worldwide [1-3]. PD clinical course is characterized by an increasing worsening of motor symptoms, which become less responsive to treatments, with the concomitant emergence of motor complications (e.g. dyskinesia, motor fluctuations, postural instability, freezing), consistently affecting quality of life (QoL). Non-motor symptoms could also be present during the whole disease course as prodromal symptoms [4, 5].

PD is caused by a reduced dopamine release due to the loss of dopaminergic neurons in the pars compacta of the substantia nigra [4]. The role of other neurotransmitters, such as glutamate, is also gaining evidence in the development of dyskinesias [6, 7]. PD therapy's goal is to increase the post-synaptic dopamine receptor stimulation with the dopamine precursor levodopa (L-dopa) and dopamine agonists. L-Dopa is the most effective PD treatment, used in association with carbidopa, benserazide, and often with catechol- $O$ methyl-transferase inhibitors (e.g. tolcapone, entacapone) to prolong its half-life, thus increasing L-dopa availability with the inhibition of its metabolism [8]. Longterm L-dopa administration is associated with the development of motor and non-motor fluctuations and dyskinesias. Loss of tonic dopaminergic regulation, changes in dopaminergic synaptic plasticity, the relatively short L-dopa half-life, and the development of the wearing-off effect are thought to play a role in the development of these manifestations. When motor complications occur, useful treatment strategies include blocking dopamine metabolism with monoamineoxidase-B inhibitors (MAOB-Is), which can also be used in the early stages of PD as monotherapy, and the administration of amantadine, an antagonist of the glutamate $\mathrm{N}$-methylD-aspartate (NMDA) receptor, involved in the development of dyskinesias $[5,7]$.

Safinamide is a potent, selective, yet reversible MAOBI that also modulates $\mathrm{Na}^{+}$and $\mathrm{Ca}^{2+}$ channels activity and reduces stimulated glutamate release, therefore acting on both the dopaminergic deficit and on a mechanism involved in dyskinesias. Safinamide has an elimination half-life ranging between 20 and $30 \mathrm{~h}$, reaching steady-state in about 1 week, and its metabolites are inactive. A single safinamide dose of about $20-40 \mathrm{mg}$ can achieve an almost complete inhibition of MAOB (about 91\%), and at doses of $\geq 600 \mu \mathrm{g} /$ $\mathrm{kg}$ the enzyme is fully inhibited $[9,10]$. Since 2015 , safinamide is approved in Europe at 50-100 mg daily dosages for the treatment of mid- to late-stage PD with motor fluctuations, as an add-on to a stable dose of L-dopa alone or in combination with other PD medications. In 2017, safinamide was approved by the US Food and Drugs Administration (FDA) as add-on therapy to L-dopa/carbidopa in PD patients experiencing "off" episodes [7, 11, 12].

So far, two network meta-analyses on MAOB-I and dopamine agonists, including safinamide, evaluated only Unified Parkinson's Disease Rating Scale (UPDRS) total score and safety $[13,14]$. A systematic review without meta-analysis concluded that safinamide was effective and safe in increasing ON-time and ameliorating motor function [15]; a systematic review without meta-analysis concluded for the efficacy of safinamide as an adjunct to L-dopa in treating motor fluctuations [16]; and one systematic review with meta-analysis showed that overall safinamide treatment significantly improved motor symptoms and QoL of PD patients [17]. No published systematic review and meta-analysis has evaluated the efficacy and safety of safinamide in PD using multiple outcomes in relation to different dose regimens, the enrollment patients with Parkinson's disease with motor fluctuations (PDwMF) or without (PDwoMF) motor fluctuations, different concomitant dopaminergic treatment, and presenting summary of findings.

Given the complexity of PD and its treatment, the objective of this systematic review and meta-analysis is the evaluation of the efficacy and safety of safinamide administration compared to placebo in PD patients on multiple outcomes. In particular, our interest is the evaluation of motor, nonmotor, and quality of life outcomes based on different available safinamide doses, the presence (or not) of motor fluctuations, and concomitant dopaminergic treatment. 


\section{Methods}

\subsection{Search Strategy and Selection Criteria}

A literature search was performed. We included randomized controlled trials (RCTs), published until 23 December 2020, satisfying the following criteria: the diagnosis was PD; the interventional drug was safinamide, alone or in association with another PD drug; the study control was placebo, alone or in association with another PD drug. Narrative or systematic reviews, or other studies not matching the prespecified inclusion criteria were excluded. No language exclusion was applied in the research and screening process.

The following databases were searched for relevant studies using "Parkinson's disease" and "safinamide" as search terms: MEDLINE (PubMed) (1966 to 23 Dec 2020); EMBASE (Embase.com) (1974 to 23 Dec 2020); Cochrane Central Register of Controlled Trials (CENTRAL 2020, Issue 12); and Latin American and Caribbean Health Science Information Database (LILACS) (Bireme) (1982 to 23 Dec 2020). For trial databases, clinicaltrials.gov (http:// www.clinicaltrials.gov); World Health Organization (WHO) International Clinical Trials Registry Platform (apps.who. int/trialsearch) for ongoing or completed trials not yet published were searched. EU and USA regulatory agencies documents for drug approvals were also searched for missing data of published and unpublished studies.

Retrieved citations were screened independently by two pairs of authors. Full texts of potentially relevant studies were consulted for inclusion or exclusion. Disagreements were resolved by collegial discussion. All studies fulfilling inclusion criteria were included in the qualitative analysis of this systematic review. Two review authors independently extracted data with basic information of each study and results. All studies were intended to be included in metaanalysis but two were excluded for methodological and statistical reasons. For papers with unavailable data, authors were contacted after the search. No answers were received till the submission of the manuscript. The manuscript was written accordingly to the PRISMA statement.

\subsection{Data Extraction}

Data were extracted independently by two authors and discrepancies were resolved by discussion. The following information was extracted: year of publication, countries involved, recruitment period, study duration, patients age and gender, inclusion and exclusion criteria, Hoehn and Yahr stage, total patients randomized and in which treatment arm, and results of prespecified review outcomes.

\subsection{Assessed Outcomes}

The primary outcomes of this study were: daily ON-time without troublesome dyskinesia, daily OFF-time [18], and UPDRS-III during ON-time (UPDRS subsection for clinician-assessed motor evaluation). Secondary outcomes were: ON-time with troublesome dyskinesia, any scales rating dyskinesia, UPDRS-II (UPDRS subsection for motor experiences of daily living evaluation), and Parkinson's Disease Questionnaire 39 (PDQ-39), evaluating QoL. Tertiary important non-motor outcomes were dysautonomia, sleep disorders, and pain. Assessed safety outcomes were patients experiencing any serious AE (SAE), treatment discontinuations due to AEs, and dyskinesia as a reported AE.

\subsection{Assessment of Risk of Bias}

Two groups of three authors each independently assessed the risk of bias of included trials according to Cochrane Rob 2 tool, which encompasses randomization process, deviations from the intended interventions, missing outcomes data, and selection of the reported result as evaluated domains [19]. The risk of bias was assessed both at study and outcome levels. For the latter, only subjective variables were assessed since all efficacy outcomes selected are of this nature. Disagreements were resolved with collegial discussion.

\subsection{Statistical Analysis}

Meta-analysis was performed when there were at least two included studies with available data for assessed outcomes. For continuous outcomes, weighted generic inverse variance on mean difference (MD) method was used to estimate MD and 95\% confidence intervals (CI). For dichotomous outcomes, Mantel-Haenszel method was used to calculate measures of effect as odds ratios (ORs) with $95 \%$ CI. Both for continuous and dichotomous data, random effects model was applied. Analysis was performed by pooling treatment arms with the same safinamide dosage compared to placebo, and by the presence of motor fluctuations. For the evaluation of dyskinesia, included studies used a modified version of the Dyskinesia Rating Scale, which does not have a minimal clinically important difference (MCID) [20]. Thus, we used the Standardized Mean Difference (SMD) method and performed a conversion into Unified Dyskinesia Rating Scale (UdysRS) units. UdysRS has available MCID [21]. Heterogeneity was tested through $I^{2}$. Meta-analysis and creation of forest plots were performed using Rev Man 5.4 (Cochrane Community, London, UK). Sensitivity analyses were performed excluding studies administering safinamide doses according to body weight. An exploratory safety analysis 
was performed on single AEs causing treatment discontinuation. ORs and 95\% CI were estimated only if a single reported $\mathrm{AE}$ caused discontinuation in at least $2 \%$ of a treatment arm.

\subsection{Summary of Findings}

Summary of findings was performed using GRADEproGDT; GRADE was performed by two review authors independently and discrepancies were resolved by discussion, according to the GRADE Handbook [22]. MCIDs for imprecision and clinical significance evaluation were searched from literature for efficacy outcomes [23-27].

\subsection{Role of the Funding Source}

There was no funding source for this study. The corresponding author had full access to all the data in the study and had final responsibility for the decision to submit for publication.

\section{Results}

\subsection{Search Results and Study Characteristics}

We identified 307 references from literature and nine from clinical trial databases. After the removal of duplicates, 231 records were found. Of these, 216 were excluded by screening their titles and abstracts. The remaining 15 studies were examined; nine studies were excluded with reasons. Therefore, six studies met the inclusion criteria for qualitative and quantitative synthesis $(009,015,016$, MOTION, SETTLE, and ME2125-3) (Fig. 1) [28-32]. The screening process retrieved one completed study with not yet published data, NCT00605683 (MOTION). Since this trial was not published in peer-reviewed journals, data were obtained by researching regulatory agencies documents $[33,34]$. Two extensions of 015 and 016 studies (017 and 018, respectively) were excluded since new inclusion and exclusion criteria were applied to the originally randomized population for the enrollment of patients $[35,36]$.

We identified one ongoing RCT (NCT03881371) evaluating safinamide versus placebo in Chinese PD patients with
Fig. 1 PRISMA flow diagram for search strategy
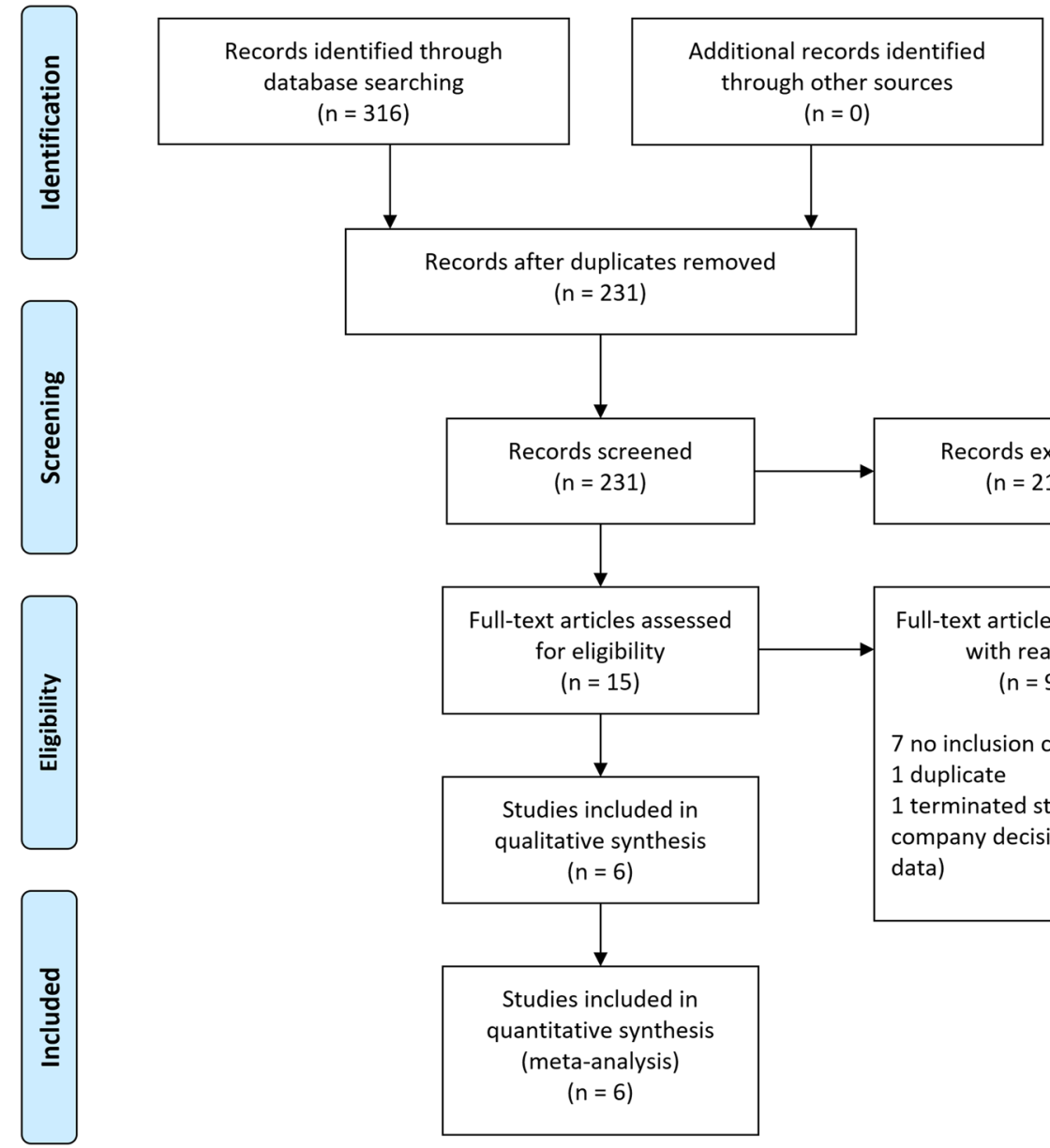
database searching $(n=316)$

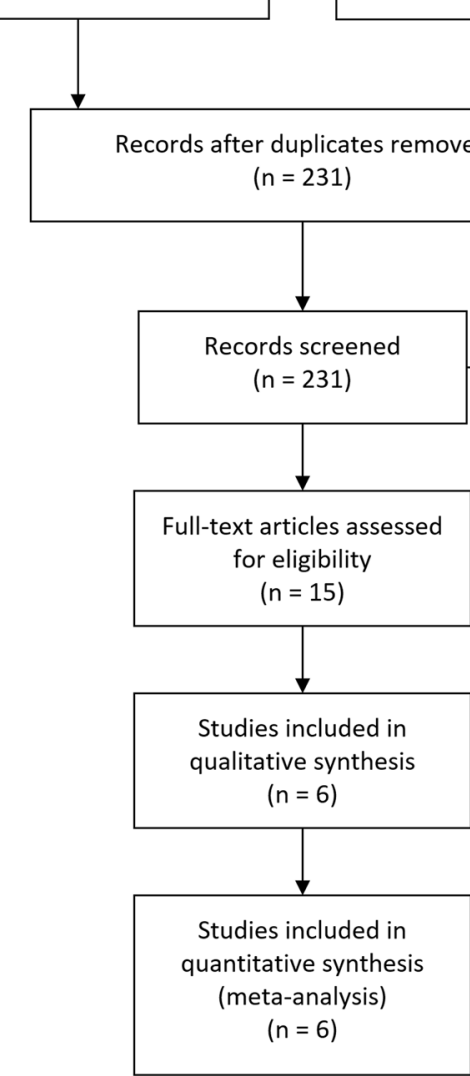

Records excluded $(n=216)$

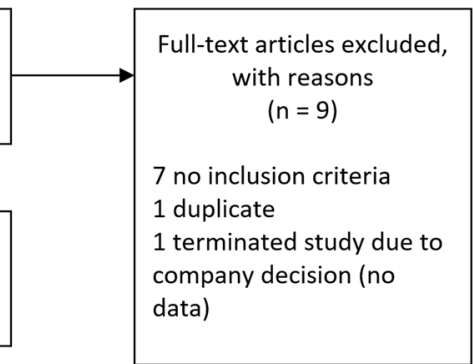


motor fluctuations while taking L-dopa, recruiting, with expected completion in September 2021. We also identified another RCT (NCT03841604) evaluating safinamide methanesulfonate in PDwMF patients and chronic pain, which is currently active, not recruiting.

Included RCTs were published between 2004 and 2020. All studies compared safinamide to placebo. Selected study characteristics are presented in Table 1.

The overall population consisted of 2729 participants with clinically diagnosed PD. A total of 1725 patients were treated with safinamide. The control population included 1004 participants, all treated with placebo. A total of 1116 patients had PDwoMF and were treated with safinamide or placebo as an add-on to a single dopamine agonist (015 and MOTION studies), whereas PDwoMF patients of 009 study received safinamide, alone or as an add-on to a single dopamine agonist, or placebo [28]. A total of 1613 patients had PDwMF (016, SETTLE, and ME2125-3 studies) and were treated with safinamide or placebo as an add-on to L-dopa with or without other PD medications. Included studies assessed the efficacy of safinamide at 50 (016, MOTION, and ME2125-3 studies), 100 (015, 016, MOTION, SETTLE, and ME2125-3 studies), and 200 (015 study) mg daily.

In SETTLE study, patients started with a $50 \mathrm{mg}$ dose, to be increased to $100 \mathrm{mg}$, if tolerated. Since $90.9 \%$ and $94.1 \%$ of patients in the safinamide and placebo group assumed the $100 \mathrm{mg}$ dose, respectively, we considered patients in the safinamide arm of this trial to be all treated with this dosage for analysis purposes. In 009 study, patients received safinamide according to body weight or placebo. Safinamide was administered at $0.5 \mathrm{mg} / \mathrm{kg}$ or $1.0 \mathrm{mg} / \mathrm{kg}$ daily doses. Median safinamide intake was $40 \mathrm{mg}$ (range from 20 to $40 \mathrm{mg}$ ) and $70 \mathrm{mg}$ (range from 40 to $90 \mathrm{mg}$ ) for the lower and higher dose, respectively [28]. For our meta-analysis, we considered the $0.5 \mathrm{mg} / \mathrm{kg}$ dose in the $50 \mathrm{mg}$ daily group and the 1.0 $\mathrm{mg} / \mathrm{kg}$ dose in the $100 \mathrm{mg}$ daily group. Sensitivity analyses were performed excluding this study and are detailed in the following sections.

\subsection{Risk of Bias}

All included studies were considered at low risk of bias for randomization process and deviations from intended interventions. ME2125-3 was considered at low risk of bias for missing outcome data; 009, 016, SETTLE, and MOTION studies received some concerns of risk of bias due to significant withdrawal from the study. However, reasons were balanced across treatment groups. The 015 study was considered at high risk of bias for missing outcome data due to significant withdrawal, which was unbalanced between groups and more frequent in the $200 \mathrm{mg}$ daily arm. All studies were considered at low risk of bias for measurement of the outcome domain. The 009 study was considered at high risk of bias for selection of the reported result since the prespecified statistical plan was not followed. Overall, ME2125-3 study was considered at low risk of bias, 016, MOTION, and SETTLE studies were received some concerns of risk of bias. Both 009 and 015 studies were considered at high risk of bias (Fig. S1). Besides, in 015 study, patients randomized to placebo received a mixture of safinamide and placebo tablets for a considerable period of the trial due to contamination of bulk placebo bottles with safinamide tablets. This was most frequently detected in the 8 - (78\% of patients) and $12-$ (58\% of patients) week pharmacokinetics analyses [29].

\subsection{Primary Outcomes}

Daily ON-time without troublesome dyskinesia (Fig. 2) and daily OFF-time (Fig. 3) were assessed in PDwMF patients treated with L-dopa [30-32]. Safinamide $100 \mathrm{mg}$ significantly increased ON-time without troublesome dyskinesia $\left(\mathrm{MD}=0.95 \mathrm{~h} ; 95 \% \mathrm{CI}\right.$ from 0.41 to $1.49 ; p=0.0006 ; I^{2}=$ $70 \%)$ and decreased OFF-time (MD $=-1.06$ hours; $95 \% \mathrm{CI}$ from -1.60 to $-0.51 ; p=0.0001 ; I^{2}=76 \%$ ). Safinamide $50 \mathrm{mg}$ significantly improved ON-time without troublesome dyskinesia $(\mathrm{MD}=0.90 \mathrm{~h} ; 95 \% \mathrm{CI}$ from 0.04 to $1.76 ; p=$ $\left.0.04 ; I^{2}=76 \%\right)$ and OFF-time (MD $=-0.86 \mathrm{~h} ; 95 \% \mathrm{CI}$ from -1.49 to $-0.24 ; p=0.007 ; I^{2}=65 \%$ ).

In PDwMF, safinamide showed a significant improvement in UPDRS-III during ON-time both at $100 \mathrm{mg}$ (MD $=-2.77 ; 95 \% \mathrm{CI}$ from -4.27 to $-1.28 ; p=0.0003 ; I^{2}$ $=69 \%)$ and $50 \mathrm{mg}(\mathrm{MD}=-2.93 ; 95 \% \mathrm{CI}$ from -5.16 to $-0.71 ; p=0.01 ; I^{2}=78 \%$ ) (Fig. 4). In PDwoMF, safinamide significantly improved UPDRS-III during ON-time only at $100 \mathrm{mg}$ dosage $(\mathrm{MD}=-1.84 ; 95 \% \mathrm{CI}$ from -3.19 to $-0.49 ; p=0.007 ; I^{2}=34 \%$, whereas nonsignificant improvement was observed for the $200 \mathrm{mg}$ ( $\mathrm{MD}=-0.30$; $95 \%$ CI from -2.22 to $1.62 ; p=0.76 ; I^{2}=$ not applicable) and $50 \mathrm{mg}(\mathrm{MD}=-1.29 ; 95 \% \mathrm{CI}$ from -3.28 to $0.70 ; p=$ $0.20 ; I^{2}=44 \%$ ) dosages (Fig. 4 ). The results of the sensitivity analyses on UPDRS-III performed excluding 009 study were consistent with the primary analyses (Fig. S8).

\subsection{Secondary Outcomes}

ON-time with troublesome dyskinesia was evaluated only in two studies in PDwMF [30, 32]. Nonsignificant differences were observed for both safinamide $100 \mathrm{mg}(\mathrm{MD}=0.14 \mathrm{~h}$; $95 \%$ CI from -0.03 to $0.30 ; p=0.10 ; I^{2}=0 \%$ ) and $50 \mathrm{mg}$ $\left(\mathrm{MD}=0.00 \mathrm{~h} ; 95 \% \mathrm{CI}\right.$ from -0.17 to $0.18 ; p=0.96 ; I^{2}=$ 0\%) (Fig. S2).

DRS was assessed only in PDwMF patients from 016 and SETTLE studies [30, 31]. Re-expressed SMD using UdysRS points showed no significant difference in DRS between safinamide and placebo both at $100 \mathrm{mg}$ (re-expressed SMD = 


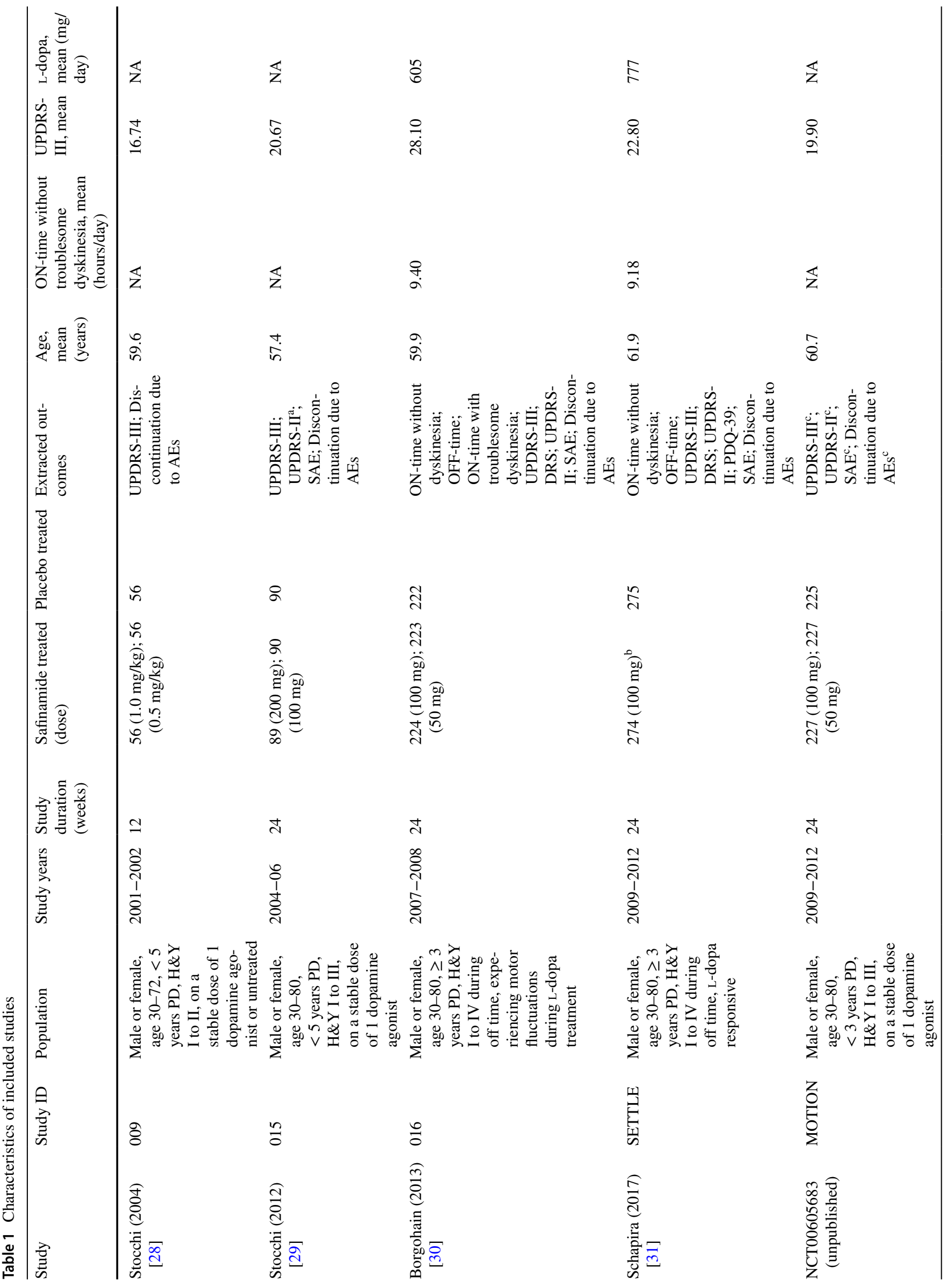




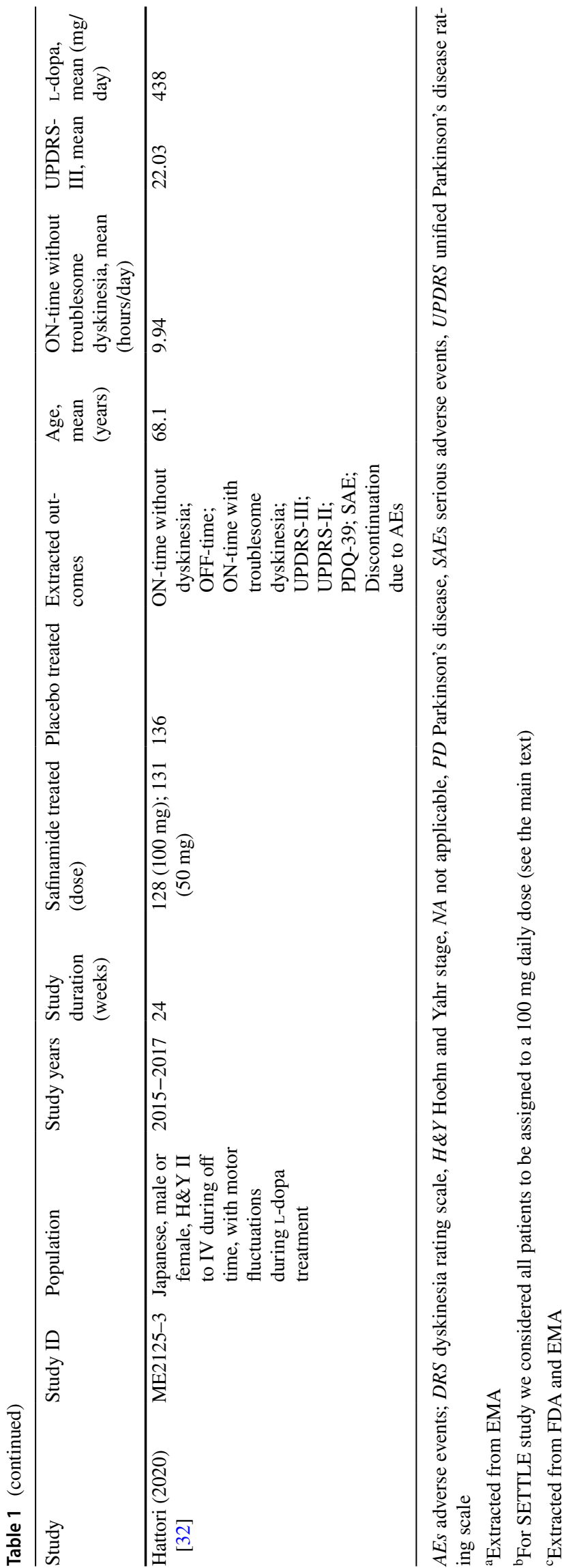

$0.42 ; 95 \%$ CI from -4.34 to $5.04 ; p=0.88 ; I^{2}=0 \%$ ) and $50 \mathrm{mg}$ (re-expressed SMD $=-1.4 ; 95 \% \mathrm{CI}$ from -7.56 to 4.76; $p=0.66 ; I^{2}=$ not applicable) (Fig. S3).

UPDRS-II data were available for all included studies except for 009 study [28]. Safinamide $100 \mathrm{mg}$ showed a significant improvement in UPDRS-II both in PDwMF (MD $=-0.65 ; 95 \% \mathrm{CI}$ from -1.03 to $-0.27 ; p=0.0009 ; I^{2}=$ $0 \%)$ and in PDwoMF (MD $=-0.55 ; 95 \%$ CI from -1.01 to $-0.09 ; p=0.02 ; I^{2}=0 \%$ ). The $50 \mathrm{mg}$ dose significantly improved UPDRS-II in PDwMF (MD $=-0.59 ; 95 \% \mathrm{CI}$ from -1.09 to $-0.09 ; p=0.02 ; I^{2}=0 \%$ ) whereas a nonsignificant difference was observed in PDwoMF for the $200 \mathrm{mg}$ $\left(\mathrm{MD}=-0.2 ; 95 \% \mathrm{CI}\right.$ from -1.12 to $0.72 ; p=0.67 ; I^{2}=$ not applicable) and $50 \mathrm{mg}(\mathrm{MD}=-0.38 ; 95 \% \mathrm{CI}$ from -0.89 to $0.13 ; p=0.14 ; I^{2}=$ not applicable) doses (Fig. S4).

PDQ-39 was available only for PDwMF patients from SETTLE and ME2125-3 studies, leading to a significant improvement in subjects treated with the $100 \mathrm{mg}$ dose (MD $=-2.32 ; 95 \%$ CI from -3.74 to $-0.89 ; p=0.001 ; I^{2}=$ $0 \%$ ), while for the $50 \mathrm{mg}$ dose the difference was nonsignificant $(\mathrm{MD}=-0.33 ; 95 \% \mathrm{CI}$ from -2.69 to $2.03 ; p=0.78$; $I^{2}=$ not applicable) (Fig. S5). RCTs assessing important non-motor symptoms were not retrieved.

\subsection{Safety}

In PDwMF, no significant differences in patients experiencing any SAE were observed in $100 \mathrm{mg}$ (OR 1.02; 95\% CI from 0.59 to $1.77 ; p=0.94 ; I^{2}=38 \%$ ) or $50 \mathrm{mg}$ (OR $0.77 ; 95 \%$ CI from 0.35 to $1.69 ; p=0.51 ; I^{2}=30 \%$ ) doses (Fig. S6). Treatment discontinuation due to AEs in PDwMF showed no significant differences for $100 \mathrm{mg}$ (OR 1.02; 95\% CI from 0.61 to $\left.1.69 ; p=0.94 ; I^{2}=0 \%\right)$ or $50 \mathrm{mg}$ (OR 0.78 ; $95 \%$ CI from 0.41 to $1.50 ; p=0.46 ; I^{2}=0 \%$ ) dosages (Fig. S7).

In PDwoMF, safinamide $200 \mathrm{mg}$ (OR 5.17; 95\% CI from 0.24 to $109.26 ; p=0.29 ; I^{2}=$ not applicable), $100 \mathrm{mg}(\mathrm{OR}$ $1.62 ; 95 \%$ CI from 0.63 to $4.13 ; p=0.31 ; I^{2}=0 \%$ ) or 50 $\operatorname{mg}\left(\right.$ OR $1.29 ; 95 \%$ CI from 0.47 to $3.51 ; p=0.62 ; I^{2}=$ not applicable) doses showed no significant differences in patients experiencing any SAE (Fig. S6). Treatment discontinuation due to AEs in PDwoMF showed no significant differences between the study groups for $200 \mathrm{mg}$ (OR 2.62; 95\% CI from 0.49 to $13.87 ; p=0.26 ; I^{2}=$ not applicable) $100 \mathrm{mg}$ (OR $0.53 ; 95 \%$ CI from 0.21 to $1.31 ; p=0.17$; $I^{2}=0 \%$ ) doses, and $50 \mathrm{mg}$ doses (OR 0.68; 95\% CI from 0.09 to $5.14 ; p=0.70 ; I^{2}=72 \%$ ) (Fig. S7). The results of the sensitivity analyses on treatment discontinuation due to AEs performed excluding 009 study were consistent with the primary analyses for $100 \mathrm{mg}$ dose. Conversely, a significant reduction in the discontinuation due to AEs was observed for safinamide $50 \mathrm{mg}$ dose (OR 0.26; $95 \% \mathrm{CI}$ from 0.07 to 0.95 ; $p=0.04 ; I^{2}=$ not applicable) (Fig. S9). For the exploratory 


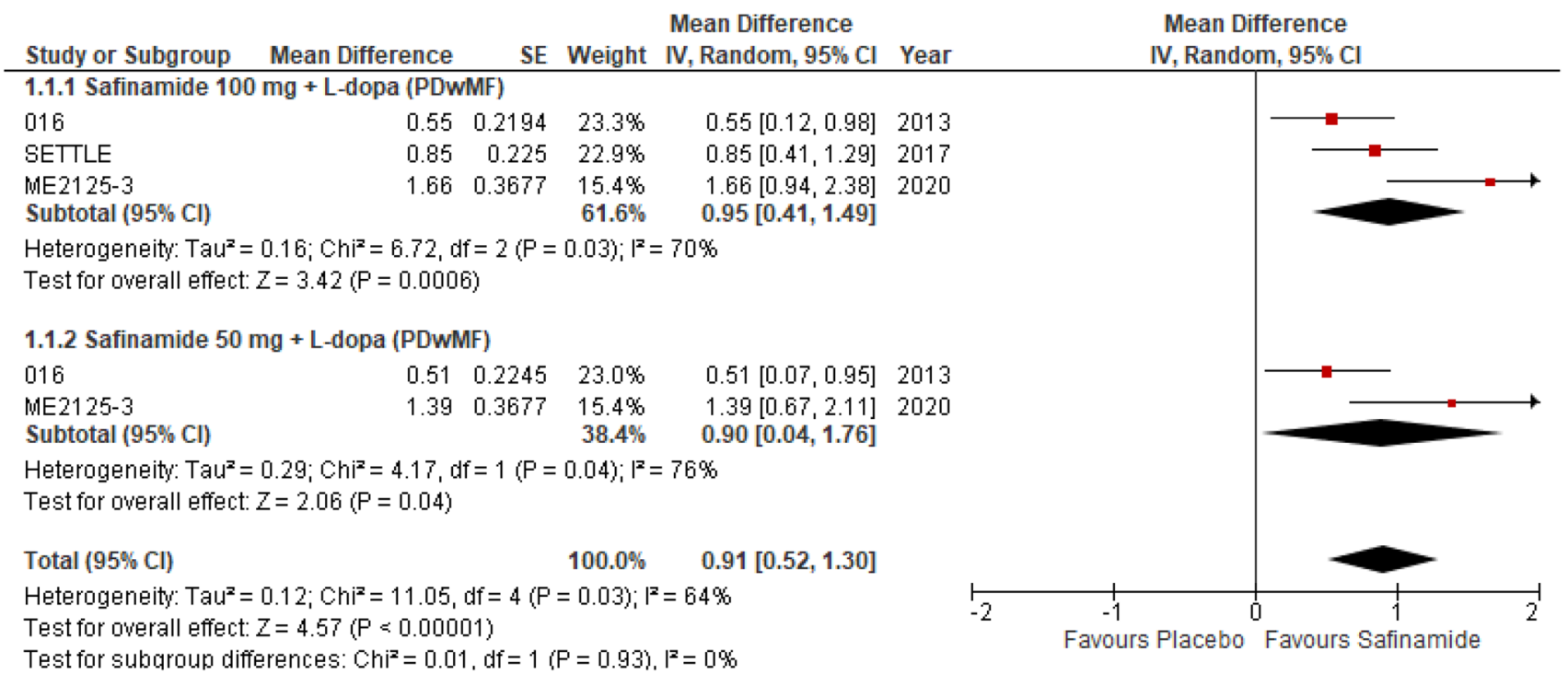

Fig. 2 ON-time without troublesome dyskinesia in PDwMF patients treated with safinamide plus L-dopa. $P D w M F$ Parkinson's disease with motor fluctuations

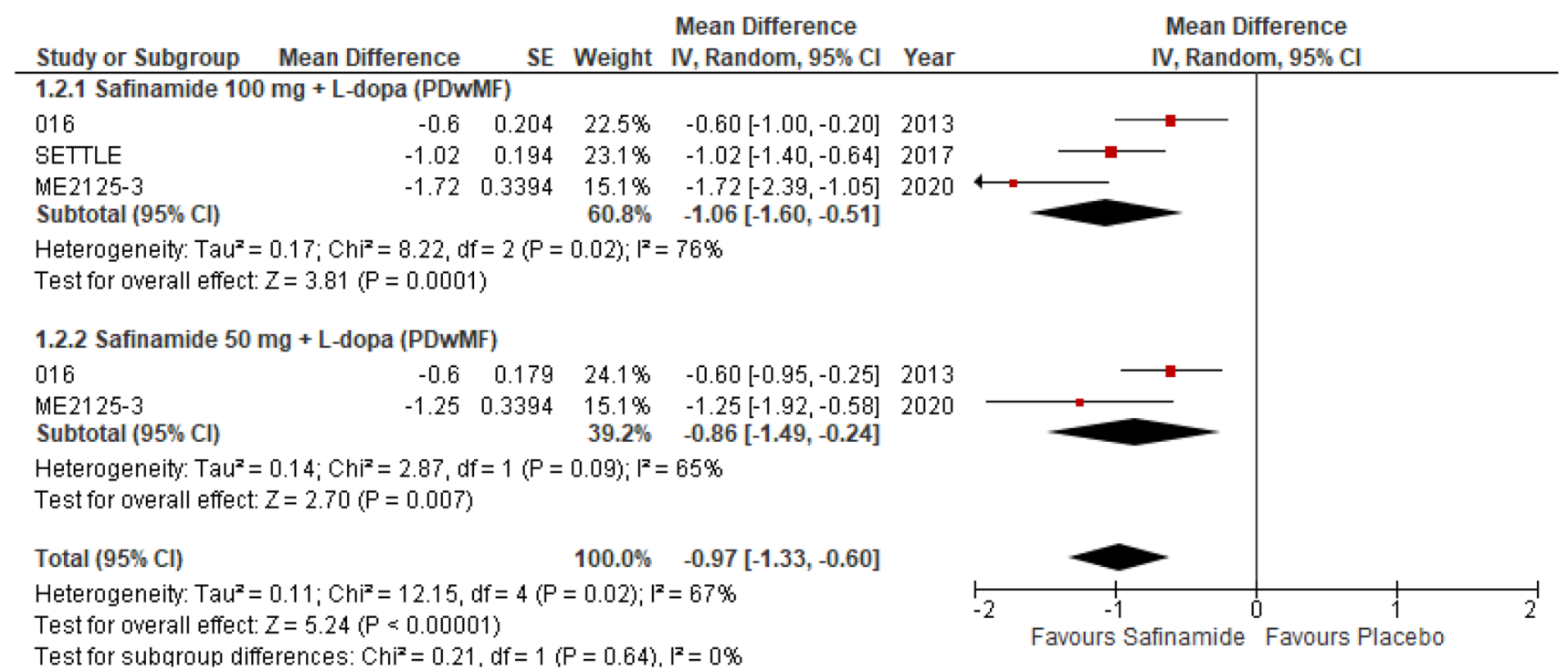

Fig. 3 OFF-time in PDwMF patients treated with safinamide plus L-dopa. PDwMF Parkinson's disease with motor fluctuations

analysis of single AEs causing treatment discontinuation, we managed to extract data only from 015, MOTION, 016, and SETTLE studies. The only AE reported more than $2 \%$ in treatment arms was dyskinesia, which caused 6, 14, and 7 discontinuations in PDwMF patients treated with safinamide $50 \mathrm{mg}$, safinamide $100 \mathrm{mg}$, and placebo, respectively. Although the tendency favored placebo, nonsignificant differences were observed between $50 \mathrm{mg}$ and $100 \mathrm{mg}$ doses and placebo (Table S1). Dyskinesia was reported as an AE mainly in 016, SETTLE, and ME2125-3 studies, including PDwMF patients taking L-dopa. Safinamide was associated with a significantly increased reporting of dyskinesia both at $100 \mathrm{mg}$ (OR 2.50; 95\% CI from 1.32 to $4.72 ; p=0.005$;
$\left.I^{2}=56 \%\right)$ and $50 \mathrm{mg}(\mathrm{OR} 2.20 ; 95 \%$ CI from 1.15 to 4.23 ; $p=0.02 ; I^{2}=22 \%$ ) in PDwMF compared to placebo. In PDwoMF, data on dyskinesia as an AE were available in a pooled analysis of 015 and MOTION studies in regulatory agencies documentation. Only one patient taking safinamide $100 \mathrm{mg}$ and one patient taking placebo reported dyskinesia, and the difference was nonsignificant (OR 1.00; 95\% CI from 0.06 to $15.96 ; p=1.00 ; I^{2}=$ not applicable) (Fig. S10). No PDwoMF reported dyskinesia while taking safinamide $200 \mathrm{mg}$ or $50 \mathrm{mg}$ doses [33].

Summary of findings with GRADE application are presented in Table 2 for safinamide $200 \mathrm{mg}$ in PDwoMF, Tables 3 and 4 for safinamide $100 \mathrm{mg}$ daily in PDwoMF and 


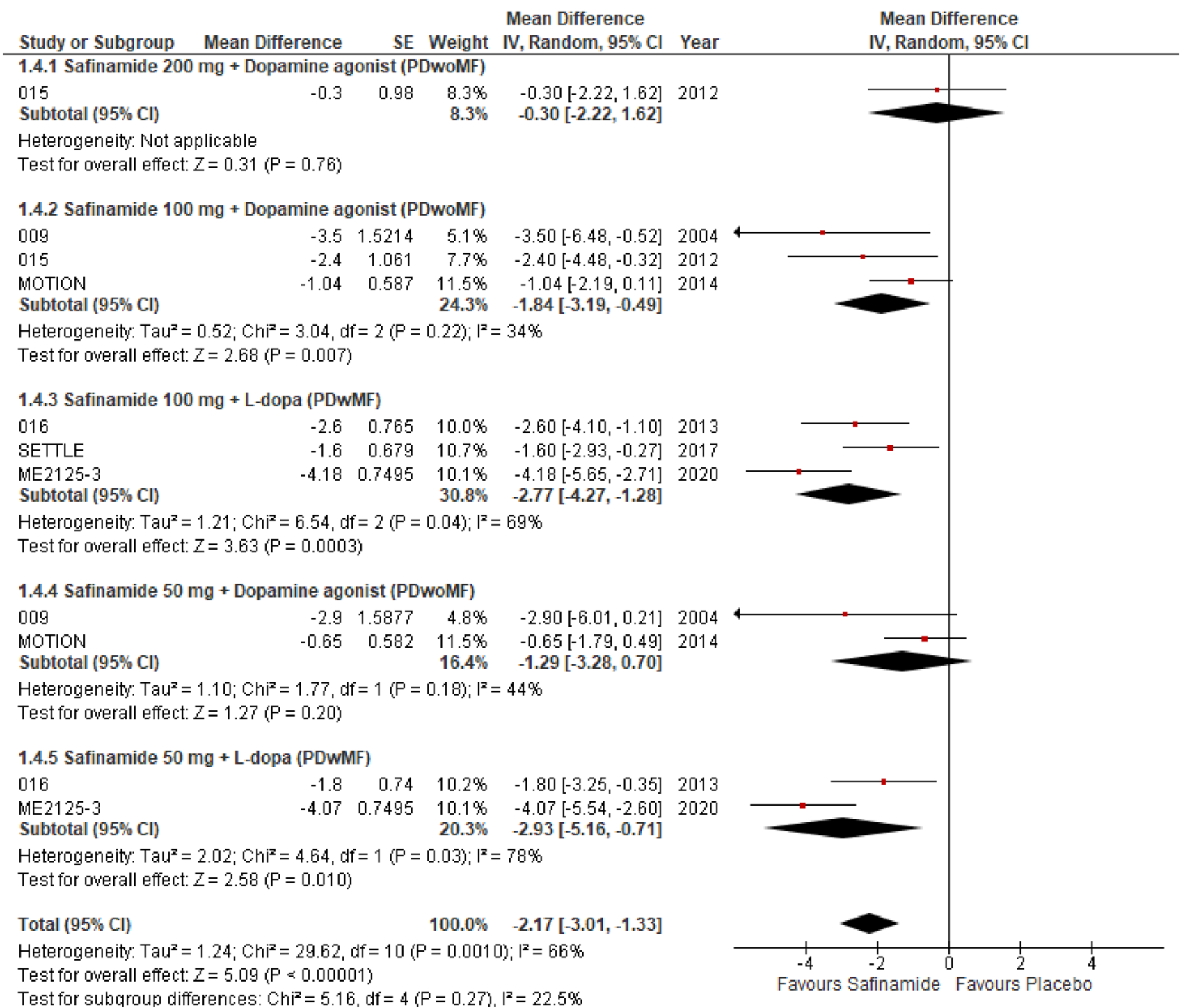

Fig. 4 UPDRS-III in PDwMF and PDwoMF patients. PDwMF Parkinson's disease with motor fluctuations, $P D w o M F$ Parkinson's disease without motor fluctuations

PDwMF, respectively, and Tables 5 and 6 for safinamide 50 mg daily in PDwoMF and PDwMF, respectively.

\section{Discussion}

In this systematic review, efficacy and safety of safinamide as add-on treatment in PDwoMF and PDwMF were assessed.

In PDwMF safinamide at $100 \mathrm{mg}$ daily dose significantly increased ON-time without troublesome dyskinesia, reduced OFF-time, and increased UPDRS-III with moderate quality of evidence. Similar results were obtained for the 50-mg dose but the evidence was lower. A significant slight reduction of UPDRS-II was observed both at $100 \mathrm{mg}$ and $50 \mathrm{mg}$ in the same patients. ON-time without troublesome dyskinesia improvement was similar to that observed in LARGO trial patients receiving rasagiline as an add-on to L-dopa compared to placebo ( $\mathrm{MD}=0.82 ; p<0.001)$ [23]. A metaanalysis showed a similar, yet slightly superior, reduction in OFF-time in patients treated with rasagiline as add-on to L-dopa $(\mathrm{MD}=-0.93 ; 95 \% \mathrm{CI}$ from -1.17 to $-0.69 ; p$ $<0.001$ ) [37]. A 2017 meta-analysis on rasagiline, both as monotherapy and as add-on to L-dopa or dopamine agonists, in PD showed that rasagiline treatment improved UPDRSIII $(\mathrm{MD}=-2.04 ; 95 \% \mathrm{CI}$ from -2.47 to $-1.61 ; p<$ $0.001)$. This result is similar to our findings in safinamidetreated patients. The same meta-analysis showed a similar but slightly higher improvement in UPDRS-II in rasagilinetreated patients [38]. A network meta-analysis of published RCTs on MAOB-I (selegiline, rasagiline, and safinamide) 
and dopamine agonists (cabergoline, pramipexole, ropinirole, and rotigotine) showed that all these compounds associated with L-dopa were superior to placebo in the response measured with UPDRS. Comparative effectiveness showed that selegiline was the most effective and safinamide less effective, immediately preceded by rasagiline [14].

Two long-term studies on safinamide provided additional data on motor outcomes in PDwMF patients. In 018 study, extending observation of 016 study up to 2 years, safinamide improved ON-time without troublesome dyskinesia and OFF-time, compared to placebo [36]. ME2125-4 was a single-arm, 52-week, safety and efficacy trial in 203 Japanese PDwMF patients. In the 194 patients efficacy population, safinamide significantly increased $\mathrm{ON}$-time without troublesome dyskinesia $(\mathrm{MD}=1.42 ; 95 \% \mathrm{CI}$ from 0.97 to 1.87) and UPDRS-III (MD $=-6.20 ; 95 \% \mathrm{CI}$ from -7.34 to -5.05$)$ and reduced OFF-time $(\mathrm{MD}=-1.40 ; 95 \% \mathrm{CI}$ from - 1.84 to - 0.96). In ME2125-4 study, the initial dose was $50 \mathrm{mg}$ and could be increased to $100 \mathrm{mg}$ during the trial. This increase was performed in 107 patients due to poor response to the lower dose, indicating that safinamide 100 mg could provide further clinical benefit [39]. In the SYNAPSES ("European multicentre retrospective-prospective cohort StudY to observe safiNAmide safety profile and pattern of use in clinical Practice during the firSt post-commErcialization phaSe") trial, a significant clinical improvement was observed in UPDRS total score (39.0\% of responders) and UPDRS-III (45.0\% of responders) at 12 months [25, 40]. Another observational study on 165 patients with PD showed significant improvements in motor and non-motor experiences of daily living assessed through the Movement Disorder Society-sponsored revision of UPDRS (MDSUPDRS) part I and II [41].

In our study, safinamide showed no significant difference in DRS compared to placebo in PDwMF patients. This observation agrees with the finding that $\mathrm{ON}$-time with troublesome dyskinesia was not modified by safinamide. However, this could be due to the use of a modified DRS version. Indeed, a study published in 2013 underlined that DRS is not sensitive to changes in dyskinesia [42]. In future RCTs

Table 2 Summary of findings for safinamide $200 \mathrm{mg}$ in PDwoMF as add-on to a single dopamine agonist

\begin{tabular}{|c|c|c|c|c|c|c|}
\hline \multirow[t]{2}{*}{ Outcomes } & \multicolumn{2}{|c|}{ Anticipated absolute effects ( $95 \% \mathrm{CI}$ ) } & \multirow{2}{*}{$\begin{array}{l}\text { Relative effect } \\
(95 \% \text { CI })\end{array}$} & \multirow{2}{*}{$\begin{array}{l}\text { No. of } \\
\text { participants } \\
\text { (studies) }\end{array}$} & \multirow{2}{*}{$\begin{array}{l}\text { Certainty of } \\
\text { the evidence } \\
\text { (GRADE) }\end{array}$} & \multirow[t]{2}{*}{ Comments } \\
\hline & Risk with placebo & $\begin{array}{l}\text { Risk with safina- } \\
\text { mide } 200 \mathrm{mg}\end{array}$ & & & & \\
\hline $\begin{array}{l}\text { UPDRS-III } \\
\text { assessed with: } \\
\text { UPDRS }\end{array}$ & $\begin{array}{l}\text { The mean UPDRS- } \\
\text { III was } 17.1 \\
\text { Points }\end{array}$ & $\begin{array}{l}\text { MD } 0.3 \text { Points } \\
\text { lower }(-2.22 \text {; } \\
1.62)\end{array}$ & - & $179(1 \mathrm{RCT})$ & $\begin{array}{l}\bigoplus \bigcirc \bigcirc \bigcirc \\
\text { VERY LOW }\end{array}$ & $\begin{array}{l}\text { The evidence is very } \\
\text { uncertain about the } \\
\text { effect of safina- } \\
\text { mide } 200 \mathrm{mg} \text { on } \\
\text { UPDRS-III }\end{array}$ \\
\hline $\begin{array}{l}\text { UPDRS-II assessed } \\
\text { with: UPDRS }\end{array}$ & $\begin{array}{l}\text { The mean UPDRS- } \\
\text { II was } 6.9 \text { Points }\end{array}$ & $\begin{array}{l}\text { MD } 0.2 \text { Points } \\
\text { lower }(-1.12 \text {; } \\
0.72)\end{array}$ & - & 179 (1 RCT) & $\begin{array}{l}\bigoplus \bigcirc \bigcirc \bigcirc \\
\text { VERY LOW }\end{array}$ & $\begin{array}{l}\text { The evidence is very } \\
\text { uncertain about the } \\
\text { effect of safina- } \\
\text { mide } 200 \mathrm{mg} \text { on } \\
\text { UPDRS-II }\end{array}$ \\
\hline $\begin{array}{l}\text { Patients experienc- } \\
\text { ing any SAE }\end{array}$ & 22 per 1000 & $\begin{array}{l}105 \text { per } 1000 \\
(5-713)\end{array}$ & $\begin{array}{l}\text { OR } 5.17(0.24 \\
109.26)\end{array}$ & 179 (1 RCT) & $\begin{array}{l}\bigoplus \bigcirc \bigcirc \bigcirc \\
\text { VERY LOW }\end{array}$ & $\begin{array}{l}\text { The evidence is very } \\
\text { uncertain about the } \\
\text { effect of safinamide } \\
200 \mathrm{mg} \text { on patients } \\
\text { experiencing any } \\
\text { SAE }\end{array}$ \\
\hline $\begin{array}{l}\text { Treatment discon- } \\
\text { tinuation due to } \\
\text { AEs }\end{array}$ & 22 per 1000 & $\begin{array}{l}56 \text { per } 1000 \\
(11-240)\end{array}$ & $\begin{array}{l}\text { OR 2.62 (0.49; } \\
13.87)\end{array}$ & 179 (1 RCT) & $\begin{array}{l}\bigoplus \bigcirc \bigcirc \bigcirc \\
\text { VERY LOW }\end{array}$ & $\begin{array}{l}\text { The evidence is very } \\
\text { uncertain about the } \\
\text { effect of safinamide } \\
200 \mathrm{mg} \text { on treat- } \\
\text { ment discontinua- } \\
\text { tion due to any AE }\end{array}$ \\
\hline
\end{tabular}

The risk $(95 \% \mathrm{CI})$ in the intervention group is based on the assumed risk in the comparison group and the relative effect of the intervention (and its $95 \% \mathrm{CI}$ )

$A E$ adverse event, $C I$ confidence interval, $M D$ mean difference, $O R$ odds ratio, $R C T s$ randomized controlled trials, $S A E$ s serious adverse events, UPDRS unified Parkinson's disease rating scale

${ }^{a}$ Risk of bias due to the majority of PBO patients in 015 study also assumed safinamide due to contamination of bulk placebo tablet bottles and high unexplained dropout rate in the $200 \mathrm{mg}$ arm

${ }^{\mathrm{b}}$ Imprecision due limited sample not meeting the optimal information size criterion

${ }^{\mathrm{c}}$ Imprecision due to few events reported and 95\% CI that includes both substantial benefit and harm 


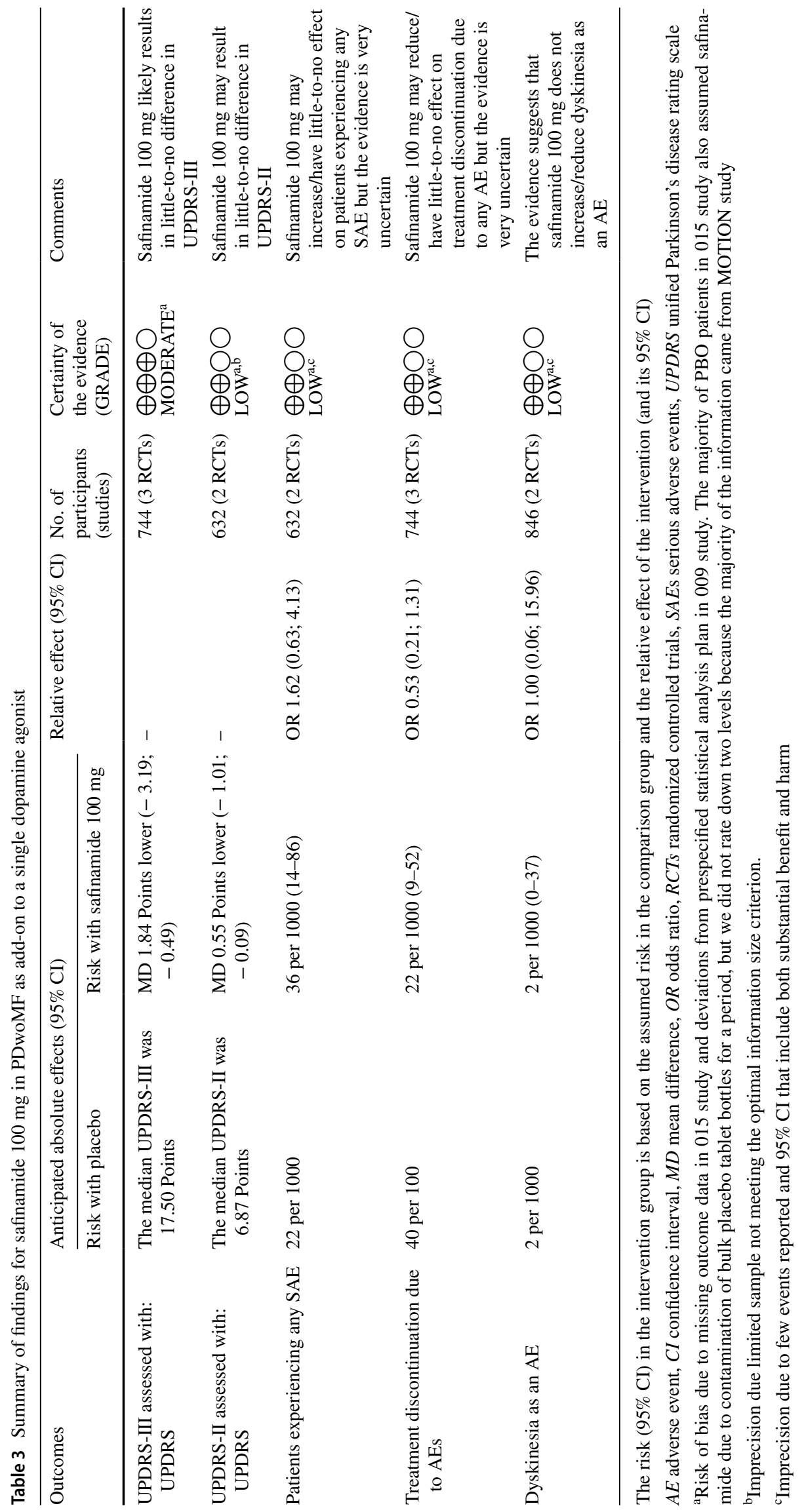




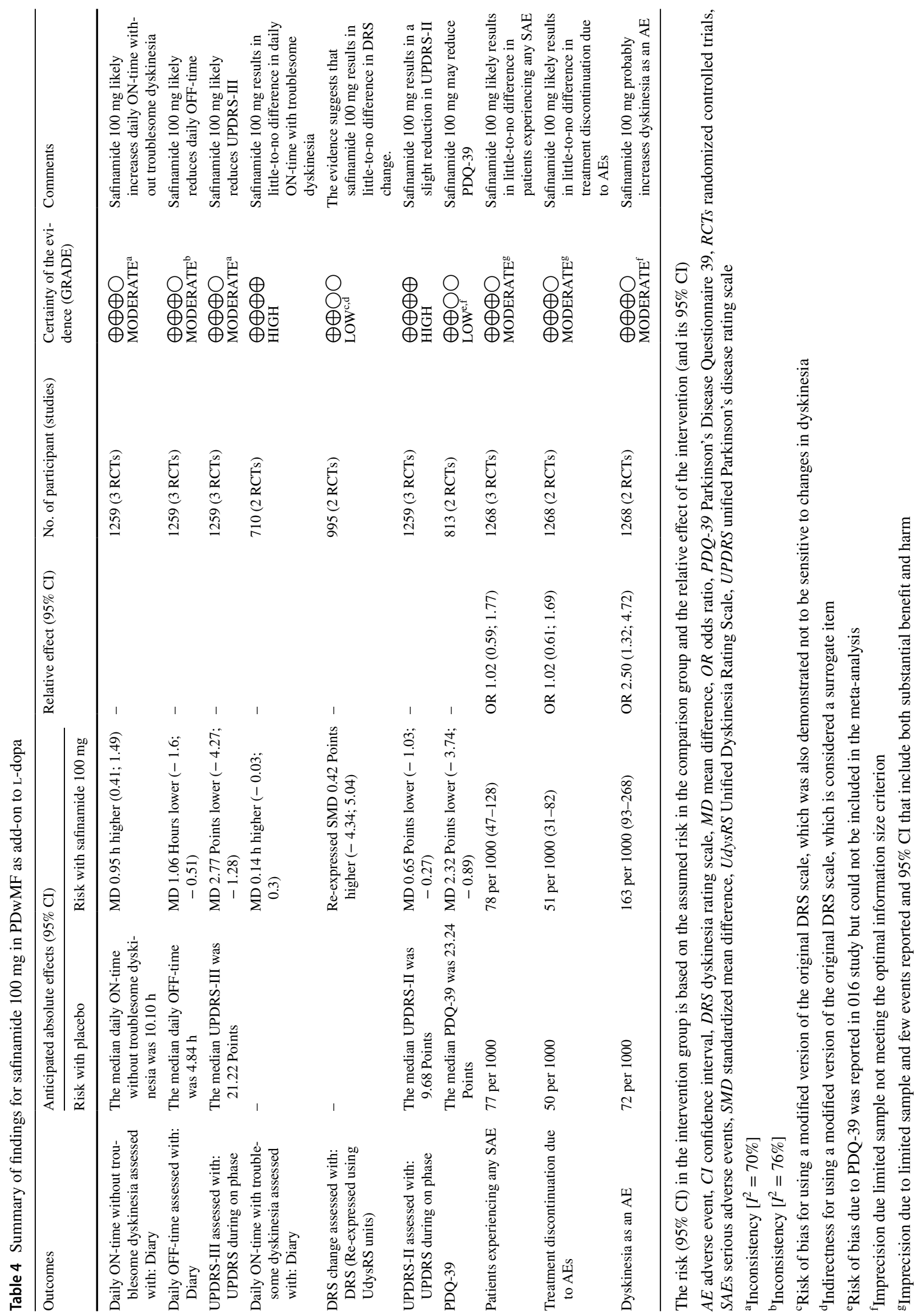




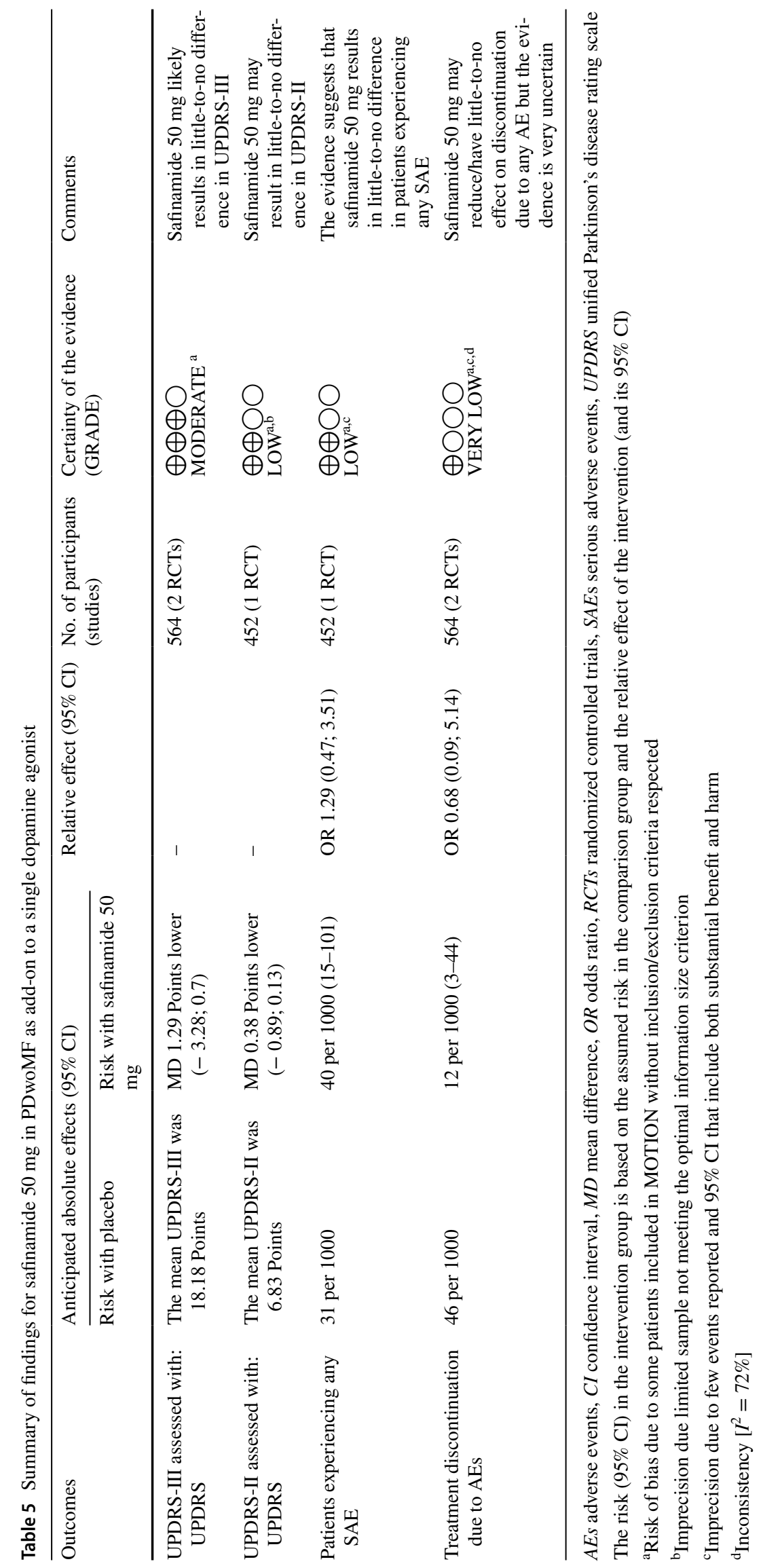




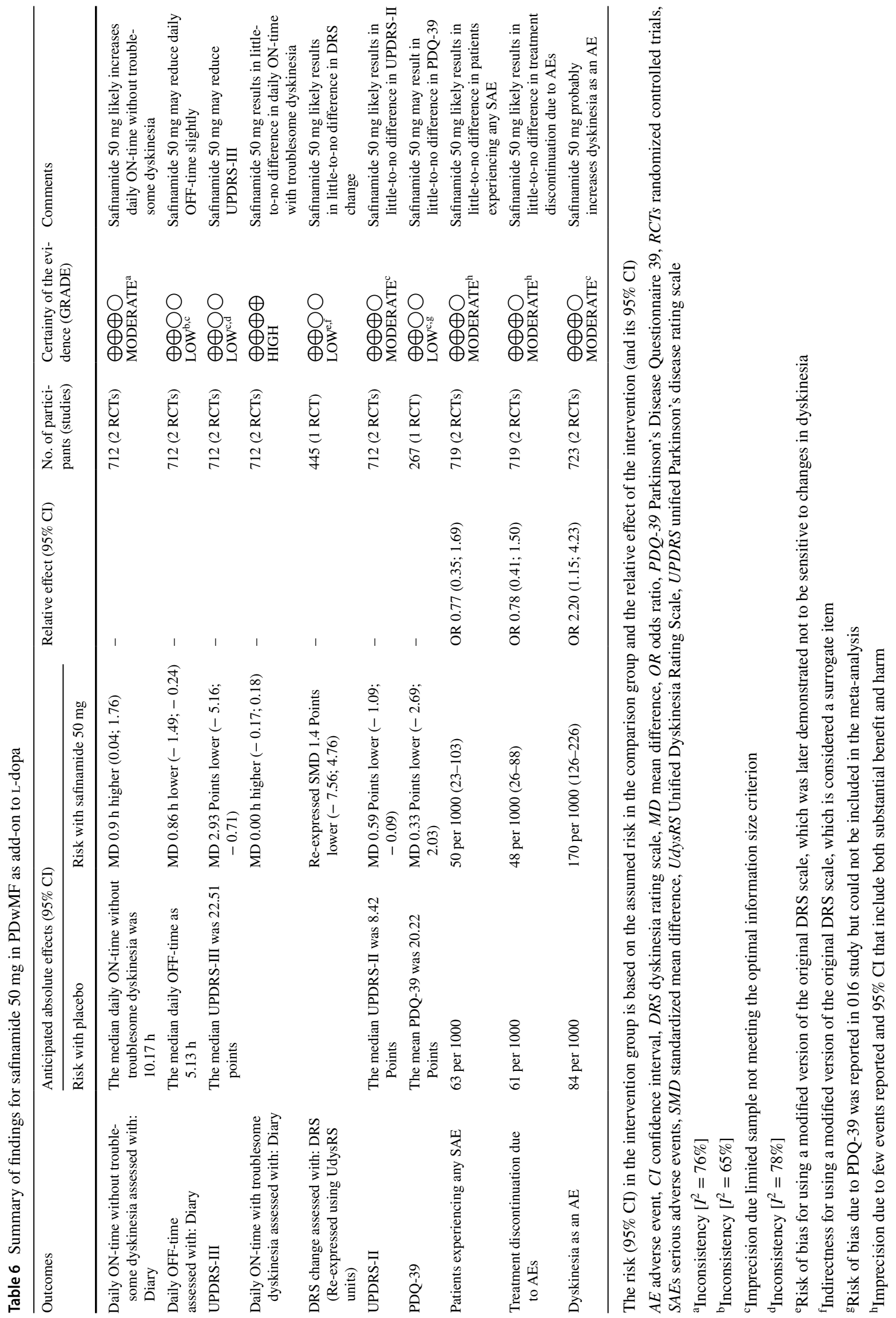


and observational studies, the use of a more robust tool to evaluate dyskinesia such as UdysRS could provide better estimates of safinamide efficacy on dyskinesias [21].

This kind of evaluation was planned in the NCT03987750 trial assessing safinamide methanesulfonate on L-dopa induced dyskinesias in PD, but the trial has been withdrawn due to changes in the drug development plan by the sponsor. The trial projected assessments included UdysRS, ON-time without troublesome dyskinesia, ON-time with troublesome dyskinesia, and MDS-UPDRS; inclusion criteria specified that participants should have at least mild severity dyskinesia and at least two 30-min periods of ON-time with troublesome dyskinesia in the two pre-randomization days [43]. In our meta-analysis $\mathrm{ON}$-time with troublesome dyskinesia showed nonsignificant differences between safinamide at 100 $\mathrm{mg}$ or $50 \mathrm{mg}$ doses compared to placebo, further corroborating the observation that safinamide does not increase dyskinesias. Nevertheless, it has to be noted that ON-time with troublesome dyskinesia is not a specific tool, such as a scale like DRS and UdysRS, for the impact and severity measurement of dyskinesias and their changes. A further reason for the observed lack of DRS reduction is that 016 and SETTLE trials enrolled fluctuating patients with an overall low DRS score [30, 31]. Besides, the post hoc analysis performed in the 016 extension study showed an improvement in DRS in patients with higher scores at baseline taking safinamide $100 \mathrm{mg}$ [36]. Considering the multiple pharmacological activities of safinamide as MAOB-I, ion-channel and glutamate release modulator $[7,11,12]$, the conduction of future RCTs and observational studies enrolling PD patients with dyskinesias, similar to NCT03987750 trial, could better clarify safinamide potential anti-dyskinetic, or at least nonpro-dyskinetic properties, which could be of great value for L-dopa-induced dyskinesias.

In PDwoMF, safinamide $100 \mathrm{mg}$ resulted in a statistically significant, yet modest and with limited clinical importance, improvement in UPDRS-III and UPDRS-II, with moderate and low quality of evidence, respectively. MDs were lower than MCIDs, but $95 \%$ CI included MCID. At $200 \mathrm{mg}$ or $50 \mathrm{mg}$, safinamide showed no significant differences in UPDRS-III and UPDRS-II. The quality of the evidence was very low for the $200 \mathrm{mg}$ dose, and moderate and low, respectively, for $50 \mathrm{mg}$. The 017 study was a 12 -month extension of 015 trial evaluating long-term efficacy and safety of safinamide compared to placebo in PDwoMF. At the end of the study, nonsignificant differences were observed in UPDRSIII and UPDRS-II between safinamide pooled treatment arms and placebo. Similarly, nonsignificant differences were observed in time-to-intervention (PD treatment escalation or discontinuation due to lack of efficacy) in the intention-totreat population, which was the primary study outcome [35].

In PDwoMF, 200, 100 or $50 \mathrm{mg}$ doses showed no significant differences in SAE reporting and treatment discontinuation with overall low quality of evidence. In PDwoMF RCTs, the risk of bias was more important than in PDwMF, in particular for 015 trial, in which for a period the majority of placebo patients assumed a mixture of safinamide and placebo tablets and there was a significant dropout rate in the 200-mg arm, being possibly related to AEs or lack of efficacy.

PDQ-39 was analyzed only in RCTs enrolling PDwMF patients treated with L-dopa, resulting in a significant reduction of the score in treated patients, indicating a likely improvement in QoL both at $100 \mathrm{mg}$ and $50 \mathrm{mg}$ dose. This outcome was assessed in MOTION, 016, SETTLE, and ME2125-3 trials, but only data from the SETTLE and ME2125-3 were available and could be used for meta-analysis. Thus, the quality of the evidence for PDQ-39 results was considered low due to imprecision and risk of bias. However, 016 study reported an improvement in PDQ-39 with safinamide $100 \mathrm{mg}$ daily [30]. Other important specific outcomes for patient's QoL, such as pain, sleep disturbances, or dysautonomia, were not present in included RCTs and should be better investigated in future clinical trials. Included studies did not assess QoL measures in primary outcomes. Future studies should consider the patient's QoL as a primary outcome, in particular with the use of patientreported outcomes, similar to the NCT03841604 trial, which is intended to evaluate safinamide methanesulfonate in pain reduction in PDwMF patients taking L-dopa.

In PDwMF, 100 or $50 \mathrm{mg}$ doses resulted in little-to-no difference in patients experiencing any SAE and treatment discontinuation due to AEs, with moderate quality of evidence. Similar results were observed in PDwoMF patients at $200 \mathrm{mg}, 100 \mathrm{mg}$, and $50 \mathrm{mg}$ doses, but the quality of the evidence was lower. Recently, the SYNAPSES trial provided further real-world data on safinamide safety [40]. After a 12-month follow-up of 1558 PD patients, 92.2\% having motor fluctuations, no differences were observed in the quality of reported AEs. Compared to pivotal 016 and SETTLE trials, the percentage of patients experiencing any $\mathrm{AE}$ was $30 \%$ lower. AEs were mild or moderate in $90.0 \%$ of patients and the most frequently reported $\mathrm{AE}$ was dyskinesia, albeit less frequently (13.7\%) compared to 016 and SETTLE studies (18.0\%). Authors report that the majority of patients complaining of dyskinesia were already presenting dyskinesias from the study beginning and experienced no further worsening. About $10.3 \%$ of patients discontinued safinamide due to AEs, a percentage superior to that observed in our meta-analysis of $100 \mathrm{mg}$ and $50 \mathrm{mg}$ doses in PDwMF. However, the SYNAPSES trial included a reallife population of PD patients, which generally present more comorbidities than patients included in RCTs [40].

In 203 PDwMF Japanese patients included in the safety population of ME2125-4 study, the safinamide safety profile was similar to 016, SETTLE, and ME2125-3 trials [39]. 
Following nasopharyngitis (20.7\%), dyskinesia was the second most frequent $\mathrm{AE}$ (17.7\%) reported. Overall, 38 dyskinesia AEs were reported, mostly in the early study period, 34 of which were resolved with dose adjustment of safinamide or other concomitant PD drugs, while 4 led to safinamide discontinuation. ON-time with troublesome dyskinesia was non-significantly changed in this study, compared to baseline $(\mathrm{MD}=-0.02 ; 95 \% \mathrm{CI}$ from -0.16 to 0.11$)$ [39].

Our meta-analysis showed an increased risk of dyskinesia as an $\mathrm{AE}$ with safinamide treatment at $100 \mathrm{mg}$ and $50 \mathrm{mg}$ doses in PDwMF patients. This observation is in contrast to the observed increase in ON-time without troublesome dyskinesia in safinamide-treated patients and the absence of difference between safinamide and placebo in ON-time with troublesome dyskinesia. However, reported dyskinesia events were mostly mild or moderate in severity and all patients had PDwMF and were taking L-dopa.

Previously, some authors hinted at the possibility of a disease-modifying role of MAOB-I in PD. However, in ADAGIO trial in PDwoMF patients treated with rasagiline, this effect could not be fully ruled out [44]. Similarly, evidence of significant delay in PD progression with selegiline was not ruled out [45]. No RCTs assessing this possible effect on safinamide were found.

Safinamide monotherapy was not assessed in RCTs except in a subgroup of participants of the 009 trial. In this study, safinamide at $1.0 \mathrm{mg} / \mathrm{kg}$ daily dose was superior $(p=$ 0.016 ) to placebo in UPDRS-III response rate at 12 weeks, defined as an improvement of at least 30\% from baseline, whereas for the $0.5 \mathrm{mg} / \mathrm{kg}$ daily dose the difference was nonsignificant $(p=0.132)$. Similar results were also observed for the difference in UPDRS-III, which is included in our meta-analysis. Notably, in the subset of naïve patients treated with safinamide alone in the 009 study, no significant differences between active treatment arms were observed, compared to placebo, in UPDRS-III response rate [28, 33]. Consequently, all clinical development of safinamide was continued only as add-on treatment [33]. Indeed, a network meta-analysis on MAOB-I and dopamine agonists evaluating UPDRS responders and SAE showed that safinamide was the only drug to be ineffective compared to placebo if administered alone [14].

A possible limitation of the present analysis is the small number of patients in evaluated outcomes. This limitation is due to the choice to perform the analysis grouping on the basis of the presence of motor fluctuations, the concomitant dopaminergic treatment (dopamine agonists or L-dopa), and different safinamide doses. Estimating the real effect of different safinamide doses in different PD stages is of great importance in clinical decision making, especially because it could be useful to define which patient would achieve major benefits from a treatment. We believe this constitutes an added value of our study since a similar analysis was not performed in previous meta-analyses [17]. Additionally, and different from previous studies [17], we excluded from analyses the extension studies of 015 and 016 trials (017 and 018, respectively), since their inclusion would have duplicated a consistent part of participants. Further added values of our study are the inclusion in the meta-analysis of data from the MOTION and ME2125-3 studies, not included in previous systematic reviews [13, 14, 17], and the presentation of summary of findings with grading of the evidence. Future studies could reduce imprecision and better define the safinamide effect, in particular on dyskinesia and nonmotor symptoms. All included RCTs were company founded and registered in clinical trial databases. Although MOTION study is so far unpublished in peer-reviewed journals, data were extracted from regulatory agencies approval files, limiting the possible risk of publication bias.

\section{Conclusion}

In conclusion, the results of this systematic review and meta-analysis support safinamide treatment at $100 \mathrm{mg}$ and $50 \mathrm{mg}$ daily as an add-on to L-dopa in PDwMF patients. Evidence for safinamide efficacy in PDwoMF as an add-on to dopamine agonists is limited. Overall, safinamide showed a good safety profile at all evaluated doses, with no differences in SAE reporting and treatment discontinuation due to AEs. The evaluation of dyskinesia with different outcomes in our meta-analysis provided, at least partially, conflicting results. The possible pharmacological activity of safinamide on dyskinesia as a primary outcome should be assessed with validated scales in future RCTs and observational studies. Important non-motor symptoms should also be better investigated. Direct comparisons with other PD drugs were not retrieved and could provide further evidence of efficacy and safety. Two RCTs are currently ongoing and could provide further data for the efficacy and safety of safinamide in the future.

Supplementary Information The online version contains supplementary material available at https://doi.org/10.1007/s40261-021-01011-y.

\section{Declarations}

Funding Open access funding provided by Università degli Studi di Milano within the CRUI-CARE Agreement.

Conflict of interest Riccardo Giossi reports non-financial support for congress participation from Mylan, outside the submitted work. Prof. Francesco Scaglione reports grants from Pfizer, lecture fees from Novartis and MSD, and has served on advisory board for GSK, outside the submitted work. Other authors declare no financial or other conflicts of interest.

Ethics approval Not applicable. 
Consent to participate Not applicable.

Consent for publication Not applicable.

Availability of data and material The datasets generated during and/or analyzed during the current study are available from the corresponding author on reasonable request.

Code availability Not applicable.

Authors' contributions R.G. contributed to study conception and design, data acquisition, analysis and interpretation, and paper drafting. F.C., M.M., F.L.R., M.S., A.S., F.C. contributed to data acquisition and analysis. V.A.F. contributed to study design and data acquisition. A.P. gave critical revision for intellectual content. I.T. contributed to data analysis and interpretation, paper drafting, and gave critical revision. A.E.E. contributed to data interpretation, paper drafting, and gave critical revision for intellectual content. F.S. gave critical revision for intellectual content.

\section{Acknowledgements None.}

Open Access This article is licensed under a Creative Commons Attribution-NonCommercial 4.0 International License, which permits any non-commercial use, sharing, adaptation, distribution and reproduction in any medium or format, as long as you give appropriate credit to the original author(s) and the source, provide a link to the Creative Commons licence, and indicate if changes were made. The images or other third party material in this article are included in the article's Creative Commons licence, unless indicated otherwise in a credit line to the material. If material is not included in the article's Creative Commons licence and your intended use is not permitted by statutory regulation or exceeds the permitted use, you will need to obtain permission directly from the copyright holder. To view a copy of this licence, visit http://creativecommons.org/licenses/by-nc/4.0/.

\section{References}

1. Tysnes OB, Storstein A. Epidemiology of Parkinson's disease. Wien: Springer; 2017. p. 901-15.

2. Dorsey ER, Sherer T, Okun MS, Bloem BR. The Emerging Evidence of the Parkinson Pandemic. J Parkinsons Dis. 2018;8(s1):S3-S8. https://doi.org/10.3233/JPD-181474.

3. Global, regional, and national incidence, prevalence, and years lived with disability for 328 diseases and injuries for 195 countries, 1990-2016: a systematic analysis for the Global Burden of Disease Study 2016. Lancet (London, England). 2017;390:1211-59.

4. Kalia LV, Lang AE. Parkinson's disease. Lancet. Lancet Publishing Group; 2015. p. 896-912.

5. Charvin D, Medori R, Hauser RA, Rascol O. Therapeutic strategies for Parkinson disease: beyond dopaminergic drugs. Nat Rev Drug Discov. 2018;17(11):804-22. https://doi.org/10.1038/ nrd.2018.136.

6. Connolly BS. Lang AE. Pharmacological treatment of Parkinson disease: A review. JAMA - J. Am. Med. Assoc. American Medical Association; 2014. p. 1670-83.

7. Jenner P. Molecular mechanisms of L-DOPA-induced dyskinesia. Nat Rev Neurosci. 2008. p. 665-77.

8. Riederer P, Gerlach M, Müller T, Reichmann H. Relating mode of action to clinical practice: dopaminergic agents in Parkinson's disease. Parkinsonism Relat Disord. 2007;13(8):466-79. https:// doi.org/10.1016/j.parkreldis.2007.06.015.
9. Marzo A, Dal Bo L, Monti NC, Crivelli F, Ismaili S, Caccia C, et al. Pharmacokinetics and pharmacodynamics of safinamide, a neuroprotectant with antiparkinsonian and anticonvulsant activity. Pharmacol Res. 2004;50:77-85.

10. Müller T, Foley P. Clinical Pharmacokinetics and Pharmacodynamics of Safinamide. Clin Pharmacokinet. 2017;56(3):251-61. https://doi.org/10.1007/s40262-016-0449-5.

11. Müller T. Safinamide: an add-on treatment for managing Parkinson's disease. Clin Pharmacol. 2018;10:31-41. https://doi. org/10.2147/CPAA.S137740.

12. Grégoire L, Jourdain VA, Townsend M, Roach A, Di Paolo T. Safinamide reduces dyskinesias and prolongs L-DOPA antiparkinsonian effect in parkinsonian monkeys. Parkinsonism Relat Disord. 2013;19(5):508-14. https://doi.org/10.1016/j.parkreldis .2013.01.009.

13. Binde CD, Tvete IF, Gasemyr J, Natvig B, Klemp M. A multiple treatment comparison meta-analysis of monoamine oxidase type B inhibitors for Parkinson's disease. Br J Clin Pharmacol. 2018;84:1917-27.

14. Binde CD, Tvete IF, Gåsemyr JI, Natvig B, Klemp M. Comparative effectiveness of dopamine agonists and monoamine oxidase type-B inhibitors for Parkinson's disease: a multiple treatment comparison meta-analysis. Germany: Eur J Clin Pharmacol; 2020.

15. Hussain S, Najimi A. Efficacy and safety of safinamide as a levodopa adjunct in patients with Parkinson disease and motor fluctuations: a systematic review of randomized controlled trials. Mov Disord. 2018;33:S101.

16. Fox SH, Katzenschlager R, Lim S-Y, Barton B, de Bie RMA, Seppi K, et al. International Parkinson and movement disorder society evidence-based medicine review: update on treatments for the motor symptoms of Parkinson's disease. Mov Disord. 2018;33:1248-66.

17. Abdelalem Aziz Ahmed M. A systematic review and meta-analysis of safety and efficacy of safinamide for motor fluctuations in patients with Parkinson's disease. F1000Research. F1000 Research Ltd; 2019;8:2078.

18. Hauser RA, Deckers F, Lehert P. Parkinson's disease home diary: further validation and implications for clinical trials. Mov Disord. 2004;19:1409-13.

19. Sterne JAC, Savović J, Page MJ, Elbers RG, Blencowe NS, Boutron I, Cates CJ, Cheng HY, Corbett MS, Eldridge SM, Emberson JR, Hernán MA, Hopewell S, Hróbjartsson A, Junqueira DR, Jüni P, Kirkham JJ, Lasserson T, Li T, McAleenan A, Reeves BC, Shepperd S, Shrier I, Stewart LA, Tilling K, White IR, Whiting PF, Higgins JPT. RoB 2: a revised tool for assessing risk of bias in randomised trials. BMJ. 2019;366:14898. https:// doi.org/10.1136/bmj.14898.

20. Goetz CG, Stebbins GT, Shale HM, Lang AE, Chernik DA, Chmura TA, et al. Utility of an objective dyskinesia rating scale for Parkinson's disease: Inter- and intrarater reliability assessment. Mov Disord. 1994;9:390-4.

21. Goetz CG, Nutt JG, Stebbins GT. The Unified Dyskinesia Rating Scale: Presentation and Clinimetric Profile. Mov Disord. 2008;23:2398-403.

22. Schünemann H, Brożek J, Guyatt G, Oxman A editors. GRADE handbook for grading quality of evidence and strength of recommendations. Updated October 2013. The GRADE Working Group; 2013.

23. Rascol O, Brooks DJ, Melamed E, Oertel W, Poewe W, Stocchi $\mathrm{F}$, et al. Rasagiline as an adjunct to levodopa in patients with Parkinson's disease and motor fluctuations (LARGO, Lasting effect in Adjunct therapy with Rasagiline Given Once daily, study): a randomised, double-blind, parallel-group trial. Lancet. 2005;365:947-54.

24. Hauser RA, Auinger P. Determination of minimal clinically important change in early and advanced Parkinson's disease. Mov 
Disord [Internet]. 2011 [cited 2020 Mar 14];26:813-8. https://doi. org/10.1002/mds. 23638 .

25. Shulman LM, Gruber-Baldini AL, Anderson KE, Fishman PS, Reich SG, Weiner WJ. The clinically important difference on the unified Parkinson's Disease Rating Scale. Arch Neurol. 2010;67:64-70.

26. Pahwa R, Tanner C, Hauser R, Isaacson S, Johnson R, Felt L, et al. A clinically important difference (CID) for the Unified Dyskinesia Rating Scale (UDysRS) Total Score Change in Parkinson's Disease (PD) Patients with Dyskinesia (P2.052). Neurology. 2018;90 (15 Sup).

27. Peto V, Jenkinson C, Fitzpatrick R. Determining minimally important differences for the PDQ-39 Parkinson's disease questionnaire.

28. Stocchi F, Arnold G, Onofrj M, Kwiecinski H, Szczudlik A, Thomas A, et al. Improvement of motor function in early Parkinson disease by safinamide. Neurology [Internet]. 2004;63:746-8. https://www.cochranelibrary.com/central/. https://doi.org/10.1002/ central/CN-00504635/full.

29. Stocchi F, Arnold G, Onofrj M, Kwiecinski H, Szczudlik A, Thomas A, Bonuccelli U, Van Dijk A, Cattaneo C, Sala P, Fariello RG; Safinamide Parkinson's Study Group. Improvement of motor function in early Parkinson disease by safinamide. Neurology. 2004;63(4):746-8. https://doi.org/10.1212/01.wnl.00001 34672.44217.f7.

30. Borgohain R, Szasz J, Stanzione P, Meshram C, Bhatt M, Chirilineau D, Stocchi F, Lucini V, Giuliani R, Forrest E, Rice P, Anand R; Study 016 Investigators. Randomized trial of safinamide add-on to levodopa in Parkinson's disease with motor fluctuations. Mov Disord. 2014;29(2):229-37. https://doi.org/10.1002/ mds. 25751.

31. Schapira AH, Fox SH, Hauser RA, Jankovic J, Jost WH, Kenney $\mathrm{C}$, et al. Assessment of safety and efficacy of safinamide as a levodopa adjunct in patients with Parkinson disease and motor fluctuations: a randomized clinical trial. JAMA Neurol. 2017;74:216-24.

32. Hattori N, Tsuboi Y, Yamamoto A, Sasagawa Y, Nomoto M. Efficacy and safety of safinamide as an add-on therapy to L-DOPA for patients with Parkinson's disease: a randomized, double-blind, placebo-controlled, phase II/III study. Parkinsonism Relat Disord. 2020;75:17-23.

33. Committee for Medicinal Products for Human Use (CHMP). Assessment report Xadago. 2014. https://www.ema.europa.eu/ en/medicines/human/EPAR/xadago. Accessed 26 Feb 2021.

34. U.S. Food and Drug Administrastion. Xadago (safinamide) Tablets [Internet]. 2017. https://www.accessdata.fda.gov/drugsatfda _docs/nda/2017/207145Orig1s000TOC.cfm. Accessed 6 Mar 2020.

35. Schapira AH, Stocchi F, Borgohain R, Onofrj M, Bhatt M, Lorenzana $\mathrm{P}$, et al. Long-term efficacy and safety of safinamide as add-on therapy in early Parkinson's disease. Eur J Neurol. 2013;20:271-80.

36. Borgohain R, Szasz J, Stanzione P, Meshram C, Bhatt MH, Chirilineau D, et al. Two-year, randomized, controlled study of safinamide as add-on to levodopa in mid to late Parkinson's disease. Mov Disord. 2014;29:1273-80.

37. Mínguez-Mínguez S, Del Pozo JSG, Jordán J. Rasagiline in Parkinson's disease: A review based on meta-analysis of clinical data. Pharmacol. Res. 2013 p. 78-86.

38. Chang Y, Wang LB, Li D, Lei K, Liu SY. Efficacy of rasagiline for the treatment of Parkinson's disease: an updated meta-analysis. Ann Med. 2017;49:421-34.

39. Tsuboi Y, Hattori N, Yamamoto A, Sasagawa Y, Nomoto M. Long-term safety and efficacy of safinamide as add-on therapy in levodopa-treated Japanese patients with Parkinson's disease with wearing-off: results of an open-label study. J Neurol Sci. 2020;416:117012.

40. Abbruzzese G, Kulisevsky J, Bergmans B, Gomez-Esteban JC, Kägi G, Raw J, Stefani A, Warnecke T, Jost WH; SYNAPSES Study Investigators Group. A European Observational Study to Evaluate the Safety and the Effectiveness of Safinamide in Routine Clinical Practice: The SYNAPSES Trial. J Parkinsons Dis. 2021;11(1):187-98.

41. Impact of safinamide on non-motor and motor experiences of daily living: Primary results from the prospective, observational ProGo study-MDS Abstracts. https://www.mdsabstracts.org/abstract/ impact-of-safinamide-on-non-motor-and-motor-experiences-ofdaily-living-primary-results-from-the-prospective-observationalprogo-study/. Accessed 19 Jan 2021.

42. Goetz CG, Stebbins GT, Chung KA, Hauser RA, Miyasaki JM, Nicholas AP, et al. Which dyskinesia scale best detects treatment response? Mov Disord. 2013;28:341-6.

43. Goetz CG, Tilley BC, Shaftman SR, Stebbins GT, Fahn S, Martinez-Martin P, et al. Movement Disorder Society-sponsored revision of the Unified Parkinson's Disease Rating Scale (MDSUPDRS): scale presentation and clinimetric testing results. Mov Disord. 2008;23:2129-70.

44. Olanow CW, Rascol O, Hauser R, Feigin PD, Jankovic J, Lang A, Langston W, Melamed E, Poewe W, Stocchi F, Tolosa E; ADAGIO Study Investigators. A double-blind, delayed-start trial of rasagiline in Parkinson's disease. N Engl J Med. 2009;361(13):1268-78.

45. Turnbull K, Caslake R, Macleod A, Ives N, Stowe R, Counsell C. Monoamine oxidase B inhibitors for early Parkinson's disease. Cochrane Database Syst Rev. 2005. https://doi.org/10.1002/14651 858.CD004898.pub2. 


\section{Authors and Affiliations}

\section{Riccardo Giossi ${ }^{1,2}$ - Federica Carrara ${ }^{1} \cdot$ Martina Mazzari ${ }^{1} \cdot$ Francesco Lo Re $^{1,3} \cdot$ Michele Senatore ${ }^{1,4}$. Azzurra Schicchi $^{5}$. Federica Corrù ${ }^{1} \cdot$ Veronica Andrea Fittipaldo $^{6}$. Arianna Pani ${ }^{1} \cdot$ Irene Tramacere $^{7}$. Antonio Emanuele Elia ${ }^{8} \cdot$ Francesco Scaglione $^{1,4}$}

1 Department of Oncology and Onco-Hematology, Postgraduate School of Clinical Pharmacology and Toxicology, University of Milan, 20129 Milan, Italy

2 Neuroimmunology and Neuromuscular Diseases Unit, Fondazione I.R.C.C.S. Istituto Neurologico Carlo Besta, Milan, Italy

3 Department of Pharmacy, "S. Maria della Misericordia" University Hospital Friuli Centrale, Udine, Italy

4 Clinical Pharmacology Unit, ASST Grande Ospedale Metropolitano Niguarda, Milan, Italy

5 Pavia Poison Control Centre - National Toxicology Information Centre - Clinical and Experimental Lab,
Toxicology Unit - Istituti Clinici Scientifici Maugeri IRCCS, Pavia, Italy

6 Oncology Department, Mario Negri Institute for Pharmacological Research IRCCS, Milan, Italy

7 Department of Research and Clinical Development, Scientific Directorate, Fondazione I.R.C.C.S. Istituto Neurologico Carlo Besta, Milan, Italy

8 Parkinson and Movement Diseases Unit, Fondazione I.R.C.C.S. Istituto Neurologico Carlo Besta, Milan, Italy 